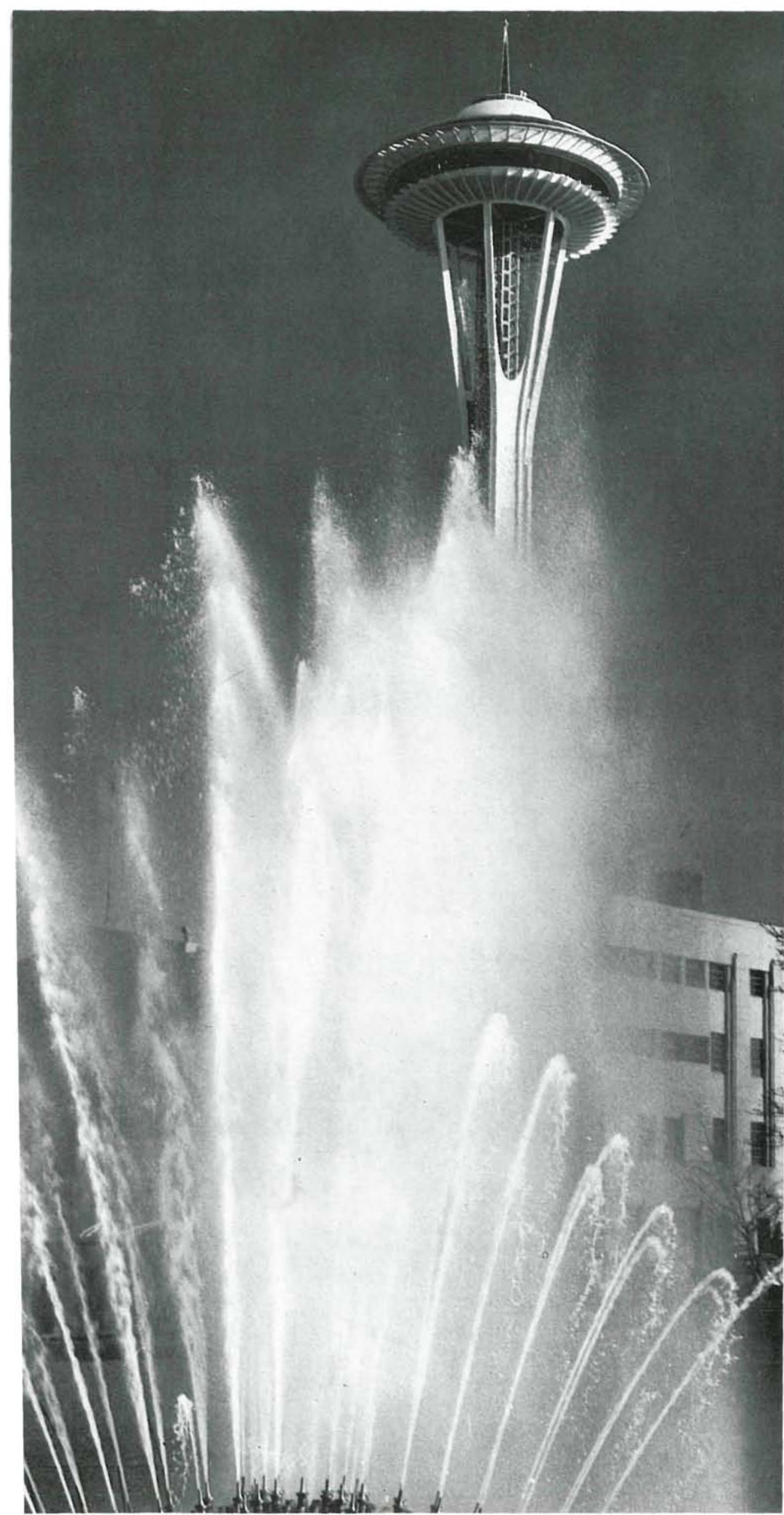

\title{
la \\ exposición mundial de Seattle
}

HARLAN H EDWARDS, Ing.

$149-12$

\section{simopsis}

El conjunto de edificios que constituyen la denominada Exposición Mun. dial de Seattle, en la ciudad de mismo nombre, Estados Unidos, ha habilitación de fondos que debian cubrir las atenciones de un proyecto de $\tan$ grandes pretensiones y mayor alcance en el orden cientifico $y$ artís. tico ha necesitado de la ayuda econó. mica federal, del Estado y de las ins-
tituciones industriales.

Cada uno de los edificios tiene una significzeión y motivo que lo caracteriza y distingue de los demás. De entre todos, destaca por su altura la chaja del Espacio, mirador $y$ antorsu capitel.

Otra obra artistica notable es la fuente luminosa, de gran contenido orna. mental y cromatico, proyectada por un grupo de arquitectos nipones.

Merece particular atención el Pabellón Científico de Estados Unidos, ya que, aparte de su aspecto formal, en êl han de exponerse los últimos avances científicos, de tal forma, que cil asimyan una vulgarización de fágeneral.

También se ha construido un teatro entaciones de ópera, conciertos, ete.

Completan el conjunto de los edificios de esta importante Exposición, una serie de instalaciones para el miento de vehículos. 


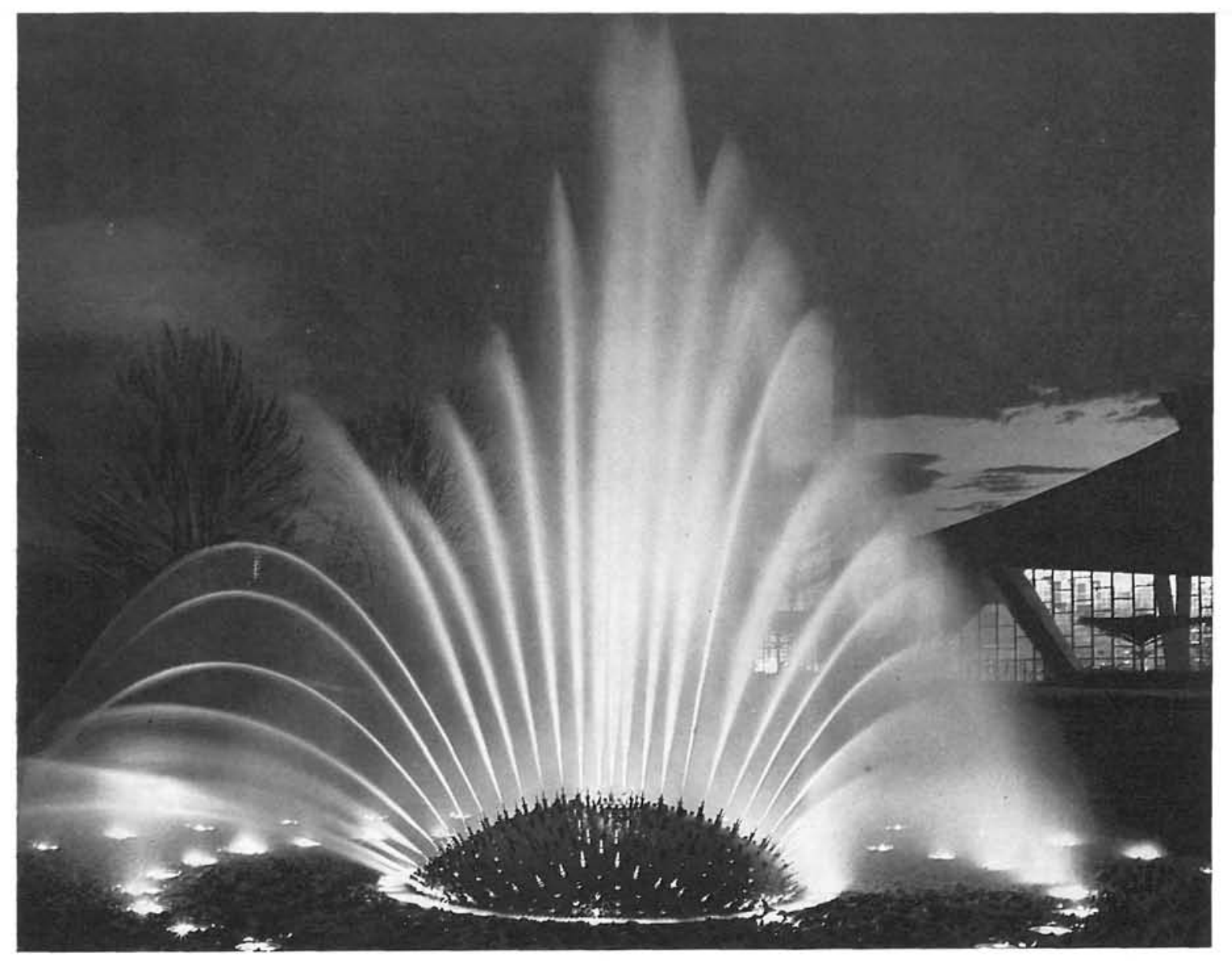

La visión y acierto de los notables arquitectos e ingenieros que han intervenido en el proyecto y ejecución de las obras de esta Exposición Mundial de Seattle, han contribuido predominantemente en la consecución de un éxito a todas luces probado. Tanto en su conjunto como en cada una de sus partes surgen manifestaciones claras de su valor intrínseco, puesto de manifiesto en el entusiasmo y aclamación popular con que ha sido aceptada por individualidades de todas partes del mundo que la han visitado. La exposición se clausuró el 21 de octubre, fecha acordada siguiendo las directivas generales establecidas por el Bureau of International Expositions, de París, Francia. Dado el entusiasmo con que la obra ha sido acogida, el numero de visitantes ha sido muy elevado y de distintas procedencias de los cinco Continentes. Una de las atracciones más destacadas la constituye el llamado Pueblo Español.

Los distintos edificios de la exposición ocupan una gran planicie, al pie de unas colinas denominadas de la Reina Ana, distantes unos 1.500 metros del centro comercial de Seattle. Desde esta zona puede contemplarse la amplia bahía de Eliott Bay and Puget Sound, con un fondo formado por escarpadas montañas cubiertas de nieve y salpicadas por varios glaciares del Parque Nacional Olympic.

La elección del lugar de emplazamiento fue decidida unánimemente por una Comisión cívica de la ciudad de Seattle y, posteriormente, ratificada por otra Comisión designada expresamente para estos fines por el Estado. El Estado de Wáshington concedió un crédito de siete millones y medio de dólares en 1957 para hacer frente a las atenciones de adquisición de terrenos y construcción de edificios, cantidad que se incrementó posteriormente, y en igual grado, por la propia ciudad de Seattle.

El fin perseguido con este proyecto tenía doble finalidad: en primer lugar, crear la Exposición Mundial de Seattle, y en segundo lugar, una vez terminada ésta, adaptarla, en sus líneas generales, a la de un Centro cívico semejante a los existentes en cualquiera de los Estados Federales. El paso definitivo para la realización de esta exposición fue dado en 1959 , habilitando un crédito de nueve millones de dólares como participación oficial y federal.

Para asegurar la mejor calidad posible y efectividad en las líneas generales del proyecto, las dos Comisiones -anteriormente citadas - conjuntaron sus esfuerzos con la aprobación de las autoridades municipales de la ciudad. El conjunto de estas Comisiones designó a un grupo de notables arquitectos-proyectistas que fijaron las premisas generales que deberían observarse en la redacción del proyecto y ejecución, recomendando se designase al conocido arquitecto Paul Thiry, de Seattle, como director de la exposición. Después de varios meses de trabajo se aprobó definitivamente el plan general que debía seguirse. El señor Thiry y sus distintos ingenieros consultores sacaron el mayor provecho posible de este plan, tanto práctica como económicamente, de la red viaria y de las distintas instalaciones, entre las que se incluyeron las líneas de electricidad y telefónicas, de tal forma que se lograse el mayor partído plástico posible. Es evidente para aquellos que pueden visitar la exposición, que una buena coordinación entre la arquitectura y la ingeniería compensa, la mayor parte de las veces, las contribuciones materiales puestas en juego. 
Las estructuras que constituyen el grupo de edificios de la exposición pueden dividirse en los siguientes grupos principales:

1. El Pabellón Cientifico de los Estados Unidos.-Las manifestaciones científicas que en él se exhiben son objeto de aclamación general como exponentes de una fineza no vista hasta la actualidad y son motivo de una tremenda atracción de las masas que la visitan.

2. El Coliseo. (Contribución económica estatal). - En él se expone lo aue se ha dado en denominar el XXI siglo Mundial. Grandes colas esperan su turno para visitar el edificio Bubbleator, formado por una especie de esfera transparente, constituyendo un ascensor con una capacidad para cien personas, que las eleva hasta llevarlas a una nube que contiene y exhibe dramáticas descripciones de ideas de la vida futura del hombre.

3. ${ }^{\circ}$ Agrupación de edificios y terrenos que forman el llamado Mundo del Comercio y de la Industria.-En esta zona muchas naciones exponen sus productos y distintos modos de vida.

4. Centro de Exposiciones de Seattle o el Mundo del Arte.-Aquí se exponen las artes más depuradas, en sus variados aspectos, elegidas entre las distintas fuentes mundiales, como se puede apreciar en diferentes fases de la exposición.

5. Auditorio para Opera y el teatro de la Comedia del Centro de Seattle.-Estos dos edificios, el primero con capacidad para 3.100 localidades y el segundo para 800, sirven, con distinción, al Mundo de Diversiones. En ellos se realizarán interpretaciones internacionales sinfónicas, ballet y conciertos.

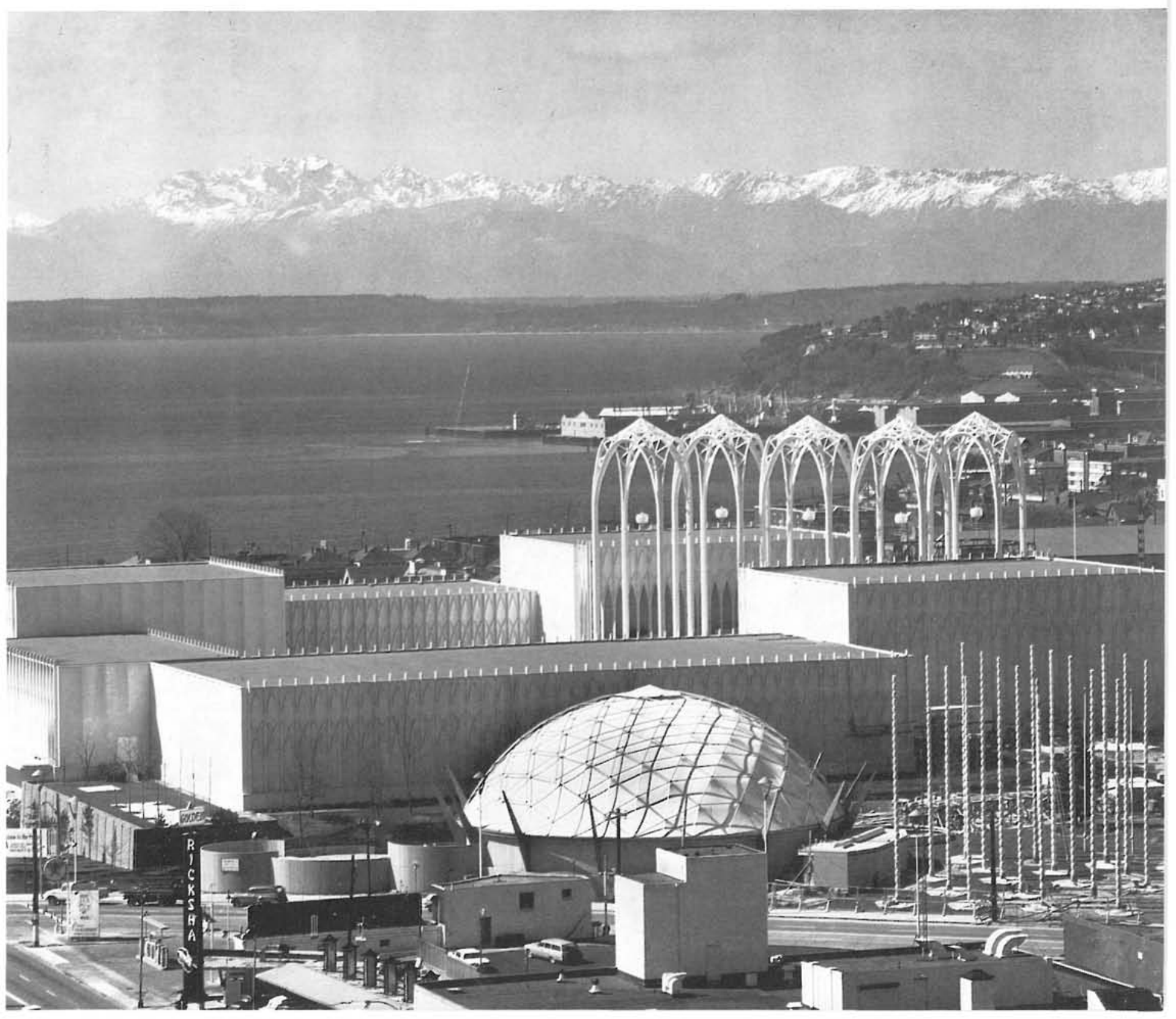


Otros edificios y extensiones de terreno se han reservado para actividades deportivas, bazares de gran colorido, restaurantes, parques y pequeños estanques.

\section{El concurso para el proyecto de ejecución del surtidor internacional}

Los dos jóvenes arquitectos nipones galardonados con la adjudicación del proyecto del surtidor internacional, han calificado dicho proyecto como una "amalgama entre la técnica espacial en el tiempo y la clásica simplicidad". Sus nombres son Hideki Shimizu y Kazuyuki Matsushita; tienen menos de treinta años de edad, y están actualmente colocados como proyeictistas en una empresa japonesa denominada Tasisei Construction Co. La ejecución de esta obra ha sido realizada por la empresa John Sellen Construction Co., que ha contado con la colaboración del arquitecto John O. Phillips, de la ciudad de Seattle.

El Jurado designado para la adjudicación del concurso fue elegido entre 265 aspirantes a jueces, procedentes de 11 naciones distintas, de entre las cuales se eligieron cinco finalistas, correspondientes a París, California, Michigán y Tokio. El agua constituye, en sí misma, el elemento escultórico En el proyecto galardonado los juegos de agua presentan formas diferentes, ya que desaparecen dentro de una cavidad de unos $30 \mathrm{~m}$ de diámetro, cuyo fondo está constituido por una roca blanca, en lugar de dejarla caer dentro del estanque convencional. Las figuras y juegos que con el agua se consiguen son controlados por medio de films electrónicos, mediante los cuales puede lograrse la repetición de las distintas figuras. También puede realizarse este control por medio de un teclado manual. La belleza y despliegue de figuras durante la noche se acentúa por medio de una luz coloreada, modulada y coordinada según las distintas figuras cambiantes del agua. El coste de construcción resultó considerablemente mayor que el previamente estimado. Se ha previsto triplicar el número de surtidores de acuerdo con las ambiciones del proyecto final y a medida que se vayan habilitando futuros créditos.

Las estructuras de esta importante Exposición Mundial de Seattle, anteriormente mencio- 


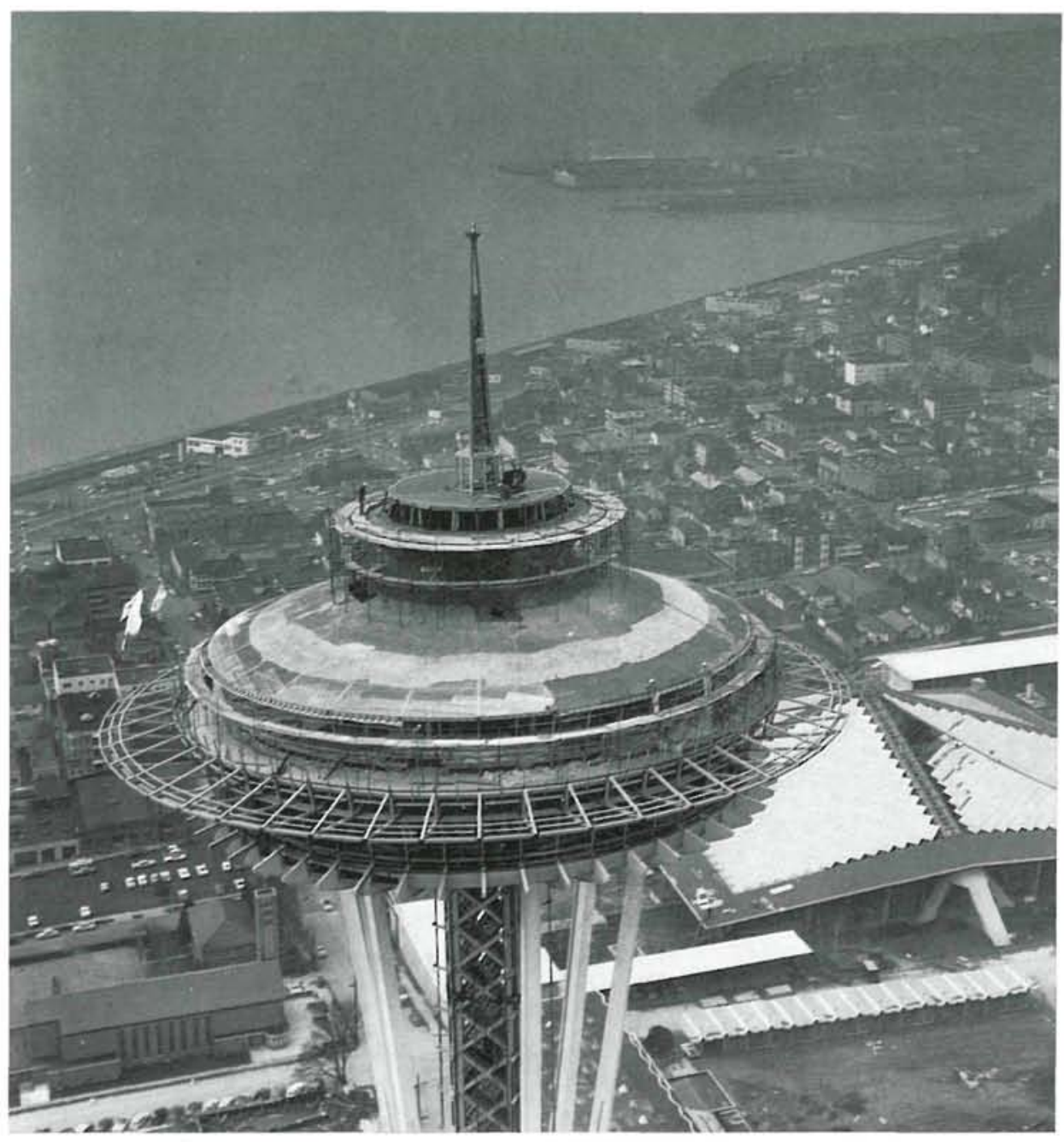

más informativo y popular.

mente, han adquirido un carácter plejo de estructuras que, actual

El tema "El hombre en la e1"a del espacio" acentúa enfáticamente "el mundo de la ciencia en el siglo XXI". Durante el período de gestación este proyecto fue entusiásticamente propuesto por las tres organizaciones científicas más importantes-la Asociación Americana para el Progreso de las Ciencias, la Academia Nacional de Ciencias y la Fundación Nacional para las Ciencias-, que constituyen el medio más efectivo para mostrar al ciudadano del mundo los grandes descubrimientos del año geofísico y sus beneficiosas aplicaciones.

Con objeto de guiar el desarrollo general, el grupo científico creó un Consejo Nacional Científico directivo, constituido por científicos de alto nivel, pertenecientes al mundo de la investigación, del gobierno y de la enseñanza. Lo que actualmente se puede contemplar constituye la descripción que los propios cientificos quieren exponer. Cada una de la exposiciones conserva la exactitud cientifica y mantiene el carácter informativo y dialogante en forma comprensible. La ciencia pura se sirve de la colaboración de la tecnología para hacer comprender mejor la historia fascinante del ciudadano de todas las edades.

nadas, constituyen, en su mayoría, proyectos de ingeniería y de arquitectura, inéditos. Durante la realización de estas artísticas obras, la atracción ha sido tal que el número de curiosos observadores se hizo interminable. De todas ellas, las principales han sido el Pabellón de la Ciencia, correspondiente a los Estados Unidos; la Aguja del Espacio; el Coliseo, del siglo XXI; el Palacio de la Opera, y la Gran Sala de Exposiciones. A todo ello deben añadirse las facilidades que han aportado el monocarril, y el garaje para estacionamiento de vehículos, obras todas ellas que hacen patente el buen gusto $y$ el cuidado general.

\section{El Pabellón \\ de la Ciencia}

\section{de los Estados Unidos}

Este conjunto de estructuras ha sido subvencionado por el Congreso con unos 10 millones de dólares para hacer frente a la adquisición de terrenos y construcción de edificios y exposiciones, a cuya aportación puede añadirse la contribución especial de otras organizaciones comerciales interesadas en este com-

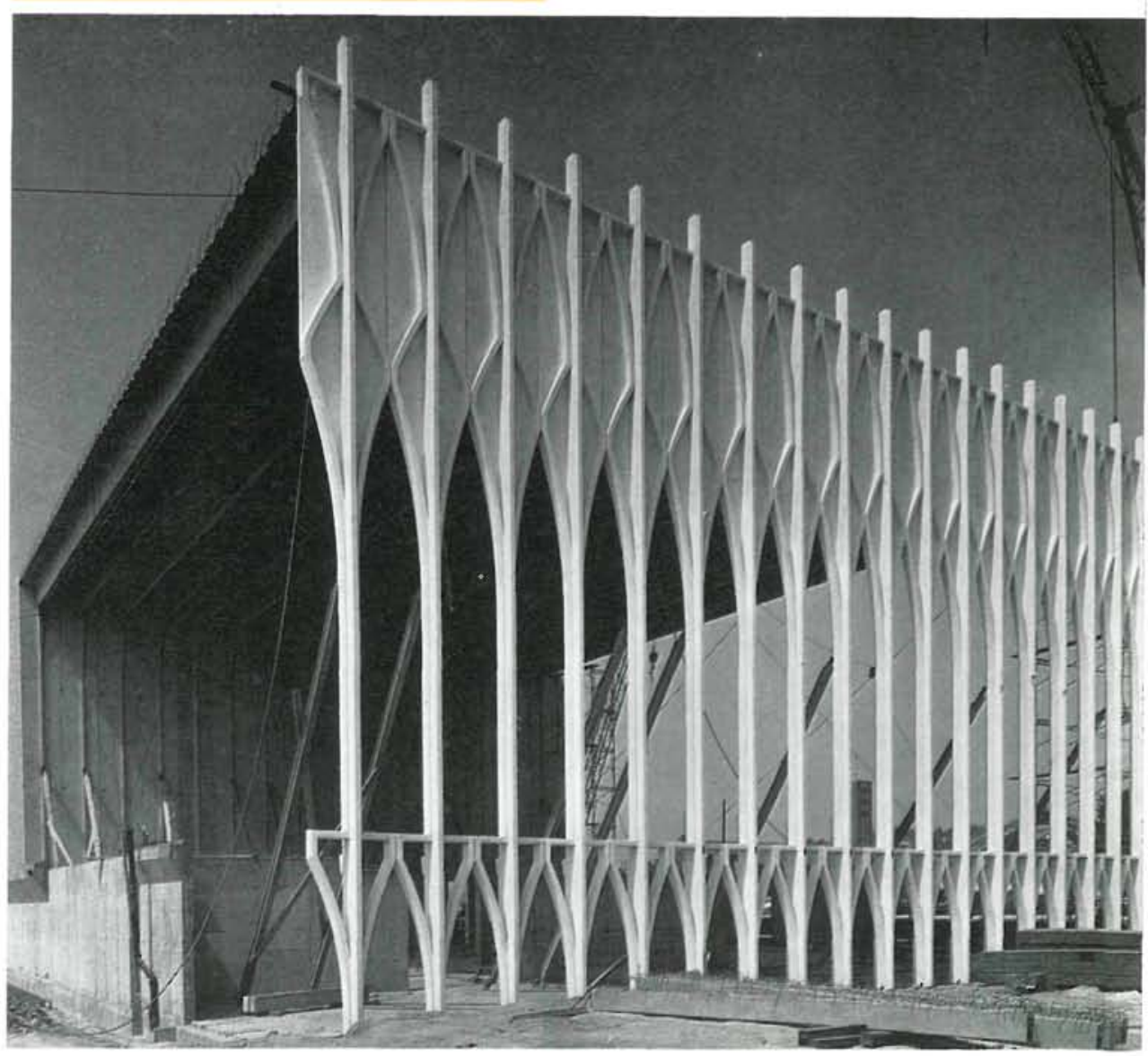




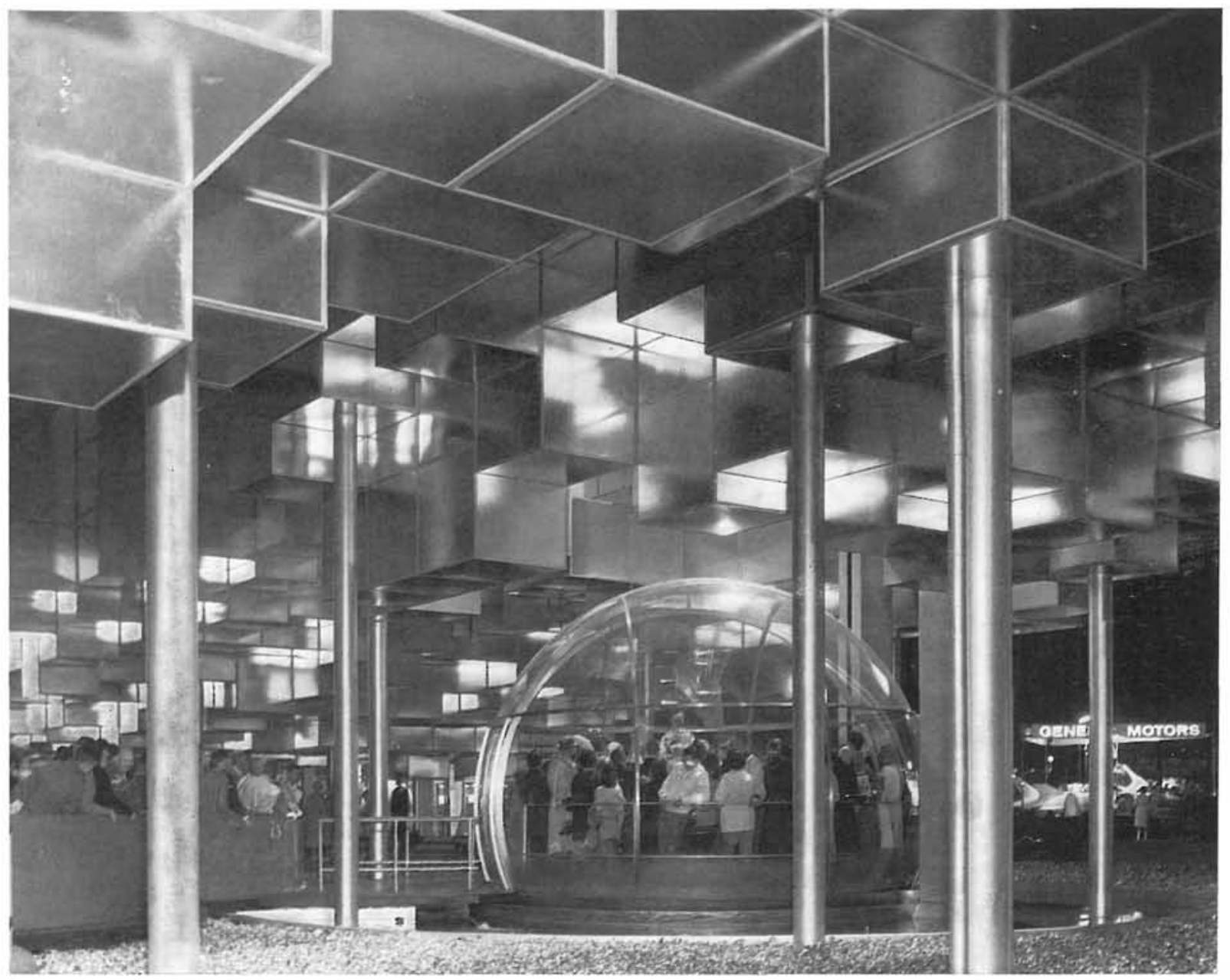

Los niños de ocho a trece años de edad también han sido tenidos en cuenta por el Laboratorio de Ciencias, que ha contribuido con 26 exposiciones distintas, cada una de ellas ilustrando principios básicos o técnicas científicas en las que estos niños pueden participar. En esta zona han sido excluidos los adultos, lo que parece han agradecido los adolescentes.

Los seis edificios que forman el Pabellón de la Ciencia se han agrupado en torno a dos estanques provistos de sus correspondientes surtidores. Bajo tierra se han construido estructuras convencionales, de hormigón armado, que sirven de apoyo a superestructuras compuestas por muros y cubiertas de elementos prefabricados, y pretensados Los paneles de los muros están revestidos con una capa de arena de cuarzo mezclada con cemento blanco. Las dimensiones de estos elementos son de 1,5 a $2,40 \mathrm{~m}$ de anchura y $7 \mathrm{~cm}$ de espesor, con montantes de $15 \mathrm{~cm}$ en el sentido transversal, constituyendo soportes o elementos de carga de hasta $16 \mathrm{~m}$ de longitud. Estos montantes soportan la carga que crean las vigas de cubierta, de sección en forma de $\mathrm{T}$, que tienen hasta $34 \mathrm{~m}$ de luz y llegan a pesar hasta $25.000 \mathrm{~kg}$. La colocación de estos elementos se ha conseguido mediante grúas montadas sobre plataformas de camiones y haciendo uso, para su fijación y unión, de soldadura y pernos, así como de elementos especiales de fijación y conexión entre dichas unidades.

Muchos de estos paneles presentan, en sus caras vistas, distintos modelos de filigranas, y se han rigidizado por medio de ligeros nervios. Esto se puede apreciar particularmente en dos de los edificios y en los cinco arcos góticos, de $30 \mathrm{~m}$ de altura, que salvan la entrada principal al patio. Las plataformas correspondientes a la parte inferior de estos arcos de entrada están constituidas por losas nervadas y pretensadas.

Uno de los edificios más espectaculares es el de Boeing Spacearium, especie de planetario que permite a los visitantes ver una trayectoria simulada de un cohete a través del espacio. Este planetario, que constituye una experiencia visual completa y en color como jamás se preparó, salva una distancia de dos billones de años-luz pero con solamente diez minutos de duración.

Esta presentación fue desarroilada con los sistemas cinerámicos aportados por la empresa Boeing Aircraft. Con este fin se utilizan simples lentes de proyección y una pantalla semiesférica, capaz de crear un área de visibilidad de $360^{\circ}$ horizontalmente y $160^{\circ}$ verticalmente.

El efecto perseguido ha sido el de envolver continuamente a los espectadores dentro de la imagen periférica.

\section{La Aguja del Espacio}

Sobre la Exposición Mundial de Seattle se levanta la Aguja del Espacio, de $185 \mathrm{~m}$ de altura, que simboliza la Era del Espacio. Esta estructura, situada en las proximidades del Pabellón de la Ciencia, de los Estados Unidos, 
presenta una forma graciosa de gavilla, de unos $152 \mathrm{~m}$ de altura y terminada por un amplio anillo, en cuyo interior se ha instalado un restaurante de $28 \mathrm{~m}$ de diámetro, que gira, imperceptiblemente, con una velocidad angular de una revolución por hora, mediante un motor eléctrico de 1 HP. El restaurante tiene una capacidad para 240 personas. Las vistas que desde sus ventanales se pueden apreciar son magníficas, pues se extienden a través de la bahía y llegan hasta las montañas cubiertas de nieve y al Parque Nacional Olympic, con el Canadá al fondo.

Por encima del restaurante se ha montado otro anillo concéntrico, cuyo espacio interior constituye un magnífico balcón, para la contemplación panorámica, con una capacidad para 600 visitantes. Por encima de esta última estructura se levanta una aguja, la cual va provista de las instalaciones interiores necesarias para suministrar gas natural a la llama con que termina la referida aguja. Esta estructura, que alcanza una altitud de $222 \mathrm{~m}$ sobre el nivel del mar, se hace visible a una distancia de $8 \mathrm{~km}$ y ha cautivado de tal manera la atención y admiración del público, que está considerada como la primera atracción de la exposición.

La construcción de esta torre, cuya base de sustentación es de hormigón armado, se realizó gracias al empleo de una grúa especialmente construida para la rápida terminación de la obra sin tener que lamentar accidente alguno. Esta grúa va montada en el interior del núcleo central de la aguja y se va prolongando, en altura, a medida que la obra adelanta, disponiendo de un mecanismo que permite sus propios movimiento y maniobra. Esta torre gigante tiene un cuerpo formado por tres pares de largos soportes que van disminuyendo de sección a medida que aumenta la altura, constituyendo así una obra maestra de simplicidad. Cada uno de los soportes consta de tres perfiles laminados, de ala ancha, de espesor reforzado y soldados a tope por las alas y rigidizados con diafragmas, que constituyen los tubos triangulares.

Con objeto de tener en cuenta los efectos del viento con grandes velocidades y las sobrecargas motivadas por movimientos sísmicos, se llevaron a cabo los correspondientes ensayos en un túnel. La resistencia prevista es superior, en una mitad, al viento de mayor velocidad que se ha registrado en la ciudad de Seattle, y dos veces superior a la carga que exigen las Ordenanzas municipales de Seattle en sus normas de construcción.

El acceso de los visitantes a la parte superior de la torre se realiza por medio de dos ascensores, con capacidad para 29 personas cada uno y velocidad de 240 metros por minuto, y un montacargas con velocidad de 120 metros por minuto. Los ascensores para el personal son cabinas con grandes cristaleras que permiten observar lo que hay al exterior; también se ha construido una escalera interior en forma de espiral, con objeto de poder complacer a cualquier visitante que desee ascender por sus propios medios.

Esta magnifica obra ha sido proyectada por John Graham Company, arquitectos e ingenieros, de la ciudad de Seattle y Nueva York.

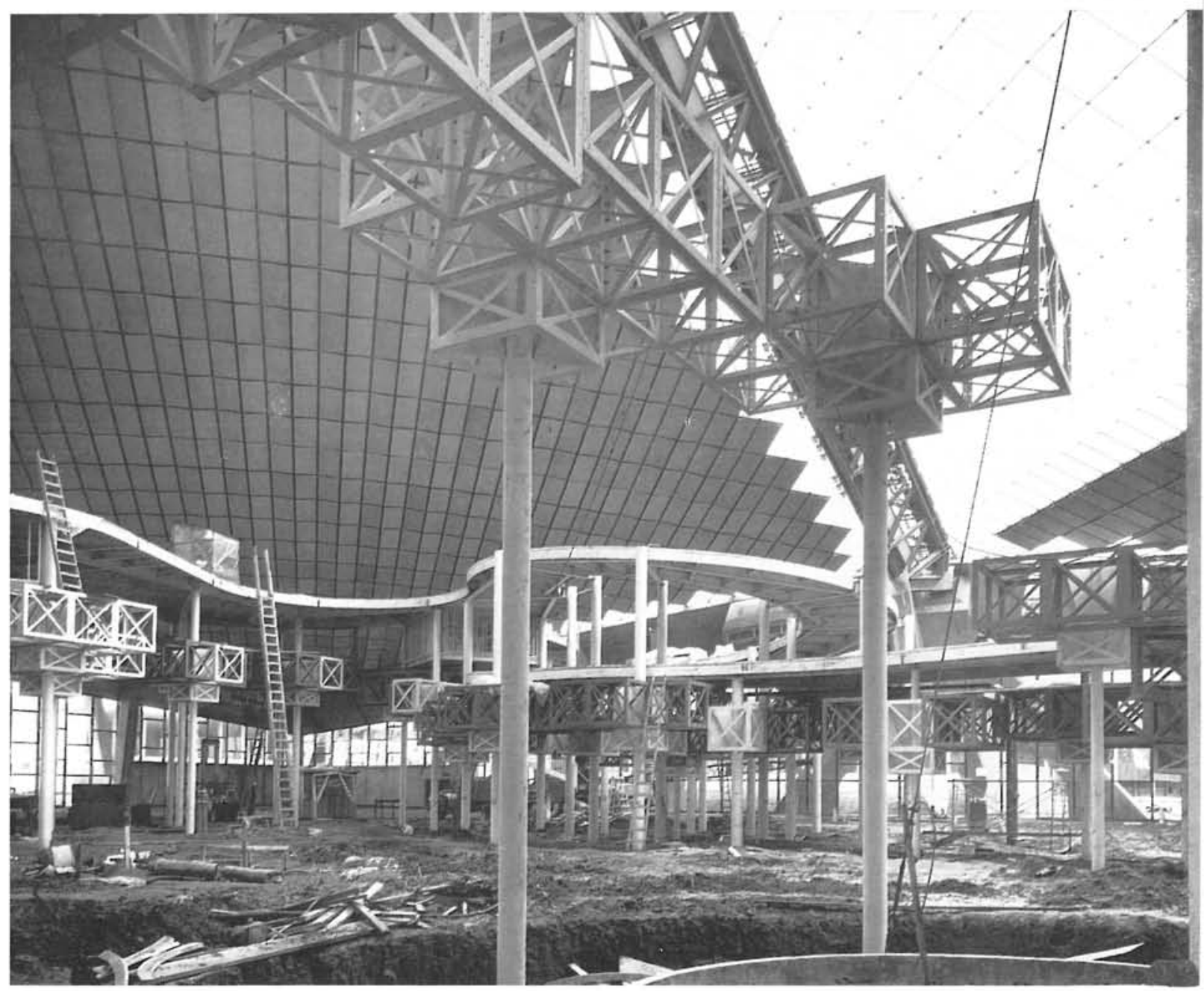




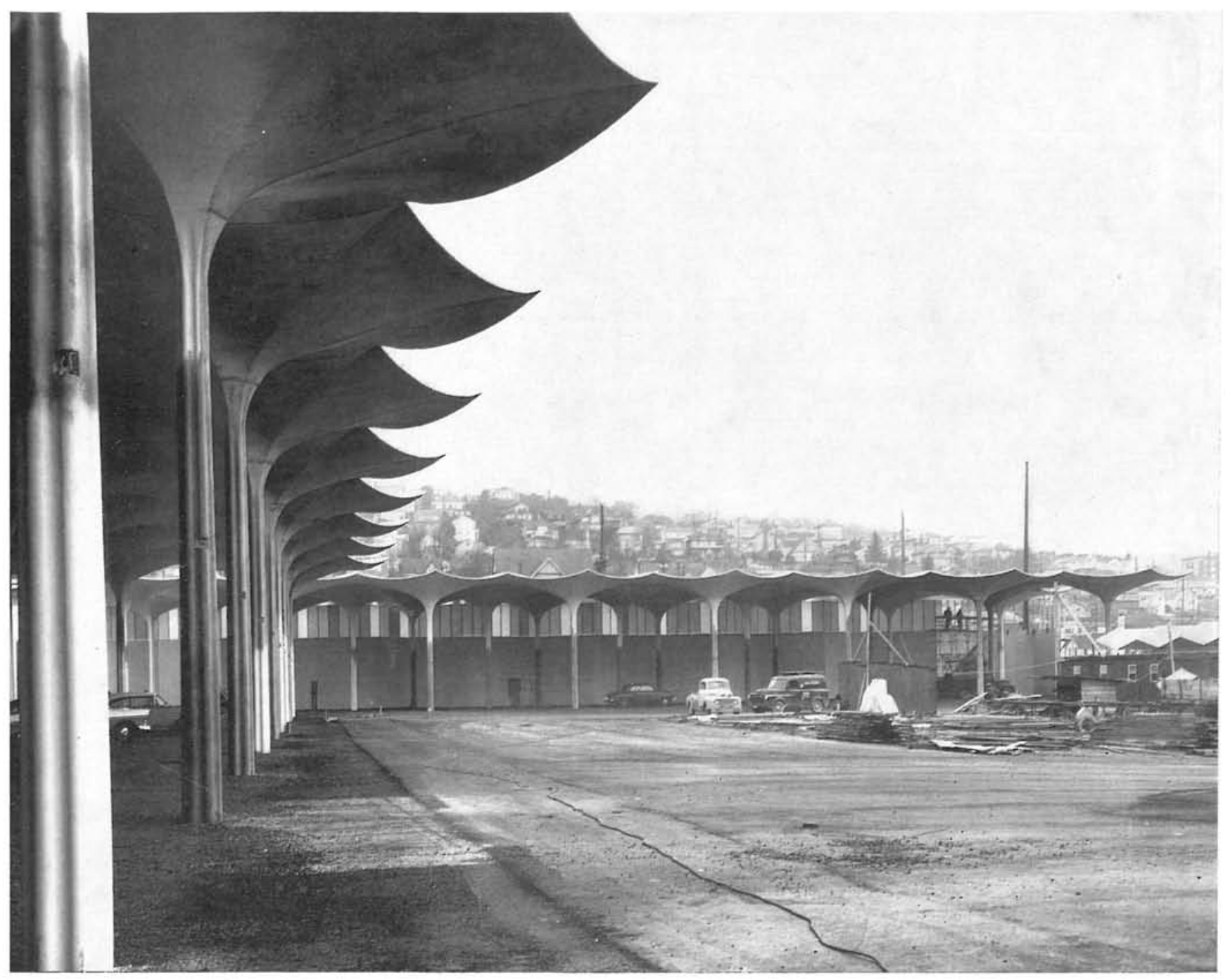

\section{El Coliseo del siglo XXI}

Este edificio, de $33 \mathrm{~m}$ de altura, se ha cubierto con paneles de aluminio, formando una superficie parabólico-hiperbólica, salvando una luz libre de $109 \mathrm{~m}$, y cuya realización ha sido posible mediante el empleo de dos celosías articuladas, de compresión, y de $3,60 \mathrm{~m}$ de anchura por $3 \mathrm{~m}$ de altura, apoyadas en estribos de hormigón formando un trípode ornamentado.

La cubierta de este edificio se ha aislado utilizando placas de aluminio anodizado, preparadas por la casa Reynolds Metals Company Co. De estos paneles de cubierta penden otros inferiores formando un ligero ángulo con objeto de lograr óptimas condiciones acústicas. Todos estos paneles se han suspendido-de una celosía y de las vigas lateralesutilizando un reticulado de cables paralelos, espaciados a $2,40 \mathrm{~m}$, y tesados posteriormente. Esta disposición ha permitido conservar la curvatura parabólico-hiperbólica entre las vigas principales. Las perimetrales son de sección triangular, hueca y postesadas.

Este edificio será dedicado, una vez terminada la exposición, a Palacio de Deportes, con una capacidad de 18 a 20.000 espectadores. Es obra del arquitecto Paul Thiry; la estructura ha sido proyectada y construida por la casa Peter Hostmark y asociados.

\section{Recinto de la Exposición del Estado de Wáshington}

Este edificio se halla en el centro del Coliseo y se ha denominado "el mundo del futuro", pues simboliza al siglo XXI. El origen de esta estructura de cubos entrelazados de aluminio se puede concentrar en la instalación denominada el "umbral de la amenaza", derivada de la ambivalencia del poder atómico, es decir, la atracción y repulsión simultánea de dicho poder, de donde se deduce la posibilidad de un doble empleo: bien constituyendo mejoras, bien en forma destructiva para el hombre y su planeta. Los visitantes acceden a esta exposición a través de una "nube de cubos", de forma general esférica, mediante un ascensor que emplea veintiún minutos en recorrer la llamada "revolución dél futuro".

\section{Zonas Internacionales de Exposición}

Los dos edificios situados al norte del Coliseo se caracterizan por su forma, semejante a la de una flor de corola abocinada, provista de paredes o láminas de $7 \mathrm{~cm}$ de espesor y de hormigón armado Las bajantes de cubierta se han situado en el interior de cada soporte y se prolongan hasta el colector. Los encofrados de las placas que constituyen 
la lámina de cubierta son de forma hexagonal, se montan y desmontan con gran rapidez, se despiezan en dos partes y han permitido un rápido progreso en la ejecución. Con el empleo de cuatro encofrados se ha podido hormigonar una lámina por día. Estos edificios y otros adjuntos, de estructura de madera, se han utilizado para exposiciones industriales y comerciales de productos extranjeros. Los arquitectos proyectistas han sido Walker \& McGough.

\section{El edificio del Centro de la Ciudad de Seattle}

Palacio de la Opera-Este nuevo teatro tiene una capacidad de 3.100 espectadores y podrá utilizarse también como sala de conciertos y otras actividades. Para su construcción se aprovechó una vieja estructura que no se amoldaba convenientemente a esta finalidad, lo que ha permitido una notable reducción en el precio total de coste, a la vez que se ha logrado mayor espacio disponible y mejoras notables. Con objeto de dar al suelo de la platea un perfil que se adaptase a la máxima visibilidad del observador, se adoptó una forma parabólica. También se habilitaron dos anfiteatros utilizando para ello, y como soporte, una viga maestra, de $36 \mathrm{~m}$ de luz, sin soporte alguno intermedio y postesada. La perfección de las condiciones acústicas y la belleza de las salas del auditorio son objeto de gran acogida, tanto por los ejecutantes como por el público.

En los alrededores del Palacio de la Opera han construido otros edificios, para exposiciones y esparcimiento, los arquitectos James Chiarelli y B. Marcus Priteca.

\section{El estacionamiento}

El problema del estacionamiento es uno de los mayores que han tenido que afrontar en el planteamiento general de esta Exposición Mundial. Su solución, y por parte de las autoridades de Seattle, ha consistido en:

1. Crear lugares de estacionamiento en las afueras de la zona en que se halla enclavada la exposición, instalando un rápido monocarril para el transporte a dichos lugares y en doble dirección.

2. Estacionamientos en el centro de la ciudad.

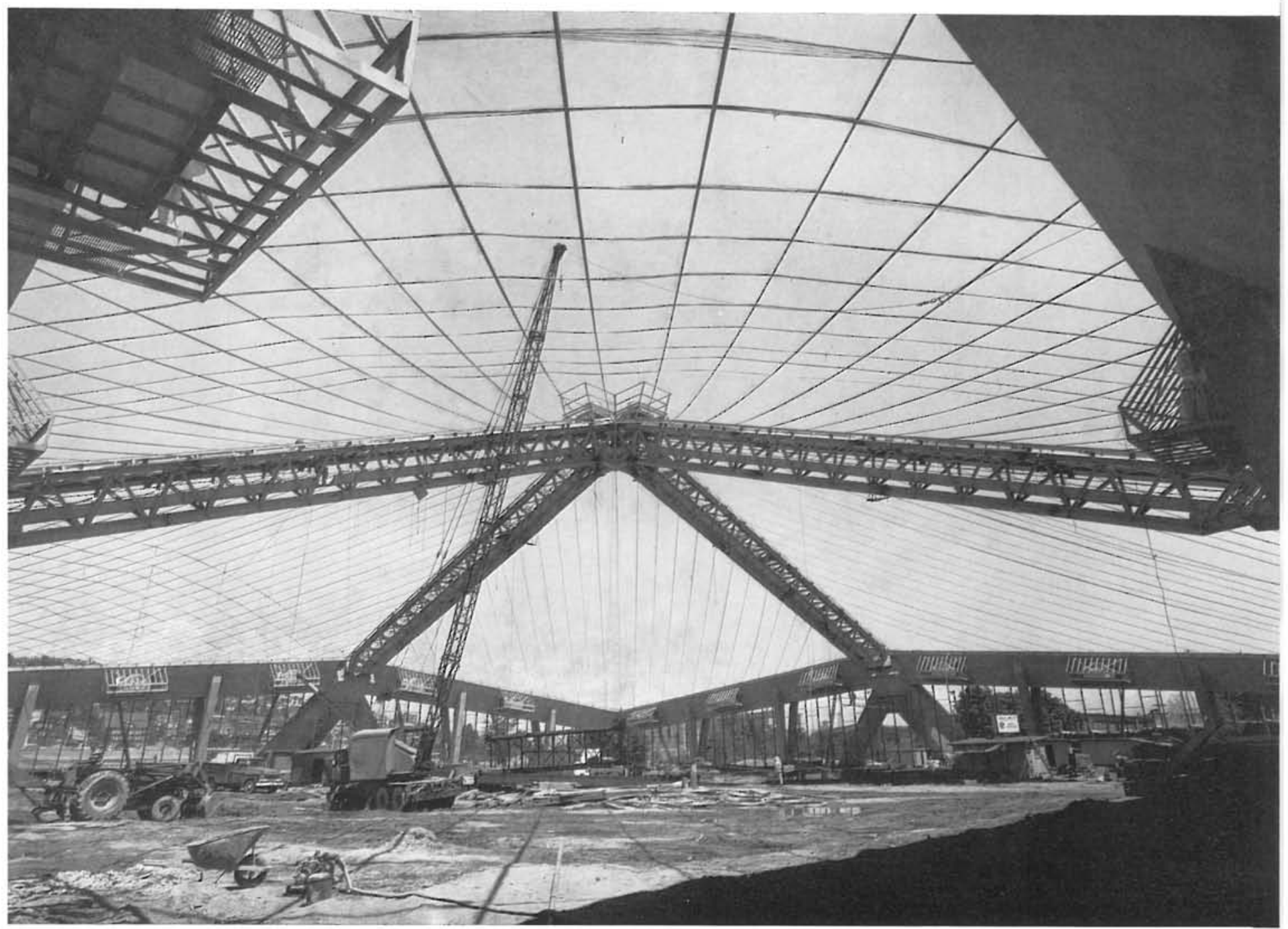



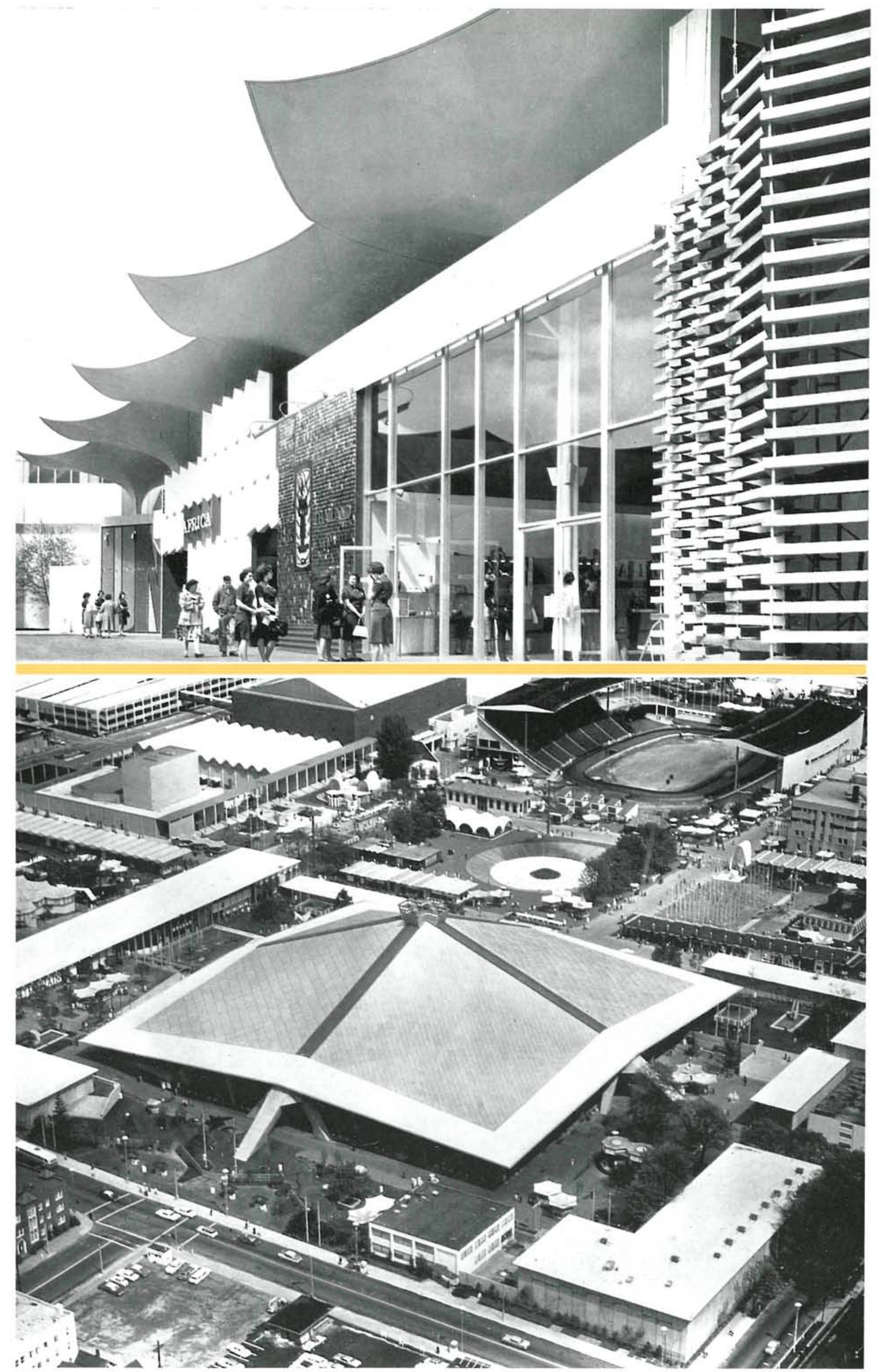
Como complemento a todo ello, y por iniciativa privada, se demolieron varios viejos edificios en un contorno, fácilmente salvado a pie, lo que permitió construir nuevos estacionamientos.

\section{El monocarril}

Este monocarril tiene un trazado de unos $1.500 \mathrm{~m}$ de longitud y una capacidad de 10.000 pasajeros por hora a una velocidad de dos minutos por viaje. Esta instalación fue proyectada por la casa sueca talación fue proyectada por la casa sueca Alweg Corporation. Para la explotación se cuenta con dos trenes eléctricos, de cuatro unidades cada uno, que se apoyan sobre vigas huecas, de hormigón pretensado, sobre las cuales se ejerce una carga máxima de 450 personas. Cada una de las dos vías tiene $90 \mathrm{~cm}$ de anchura y $150 \mathrm{~m}$ de profuncidad $y$ se han montado por tramos de $36 \mathrm{~m}$ de longitud, apoyándose sobre soportes de hormigón, troncocónicos, cuya sección tiene forma de T terminando en un cuadrado de $1,20 \mathrm{~m}$ de lado en su parte inferior, correspondiente al nivel de la calzada. Estos soportes, prefabricados a pie de obra, se colocaron-a $6,40 \mathrm{~m}$ de separacion-utilizando gruas moviles.

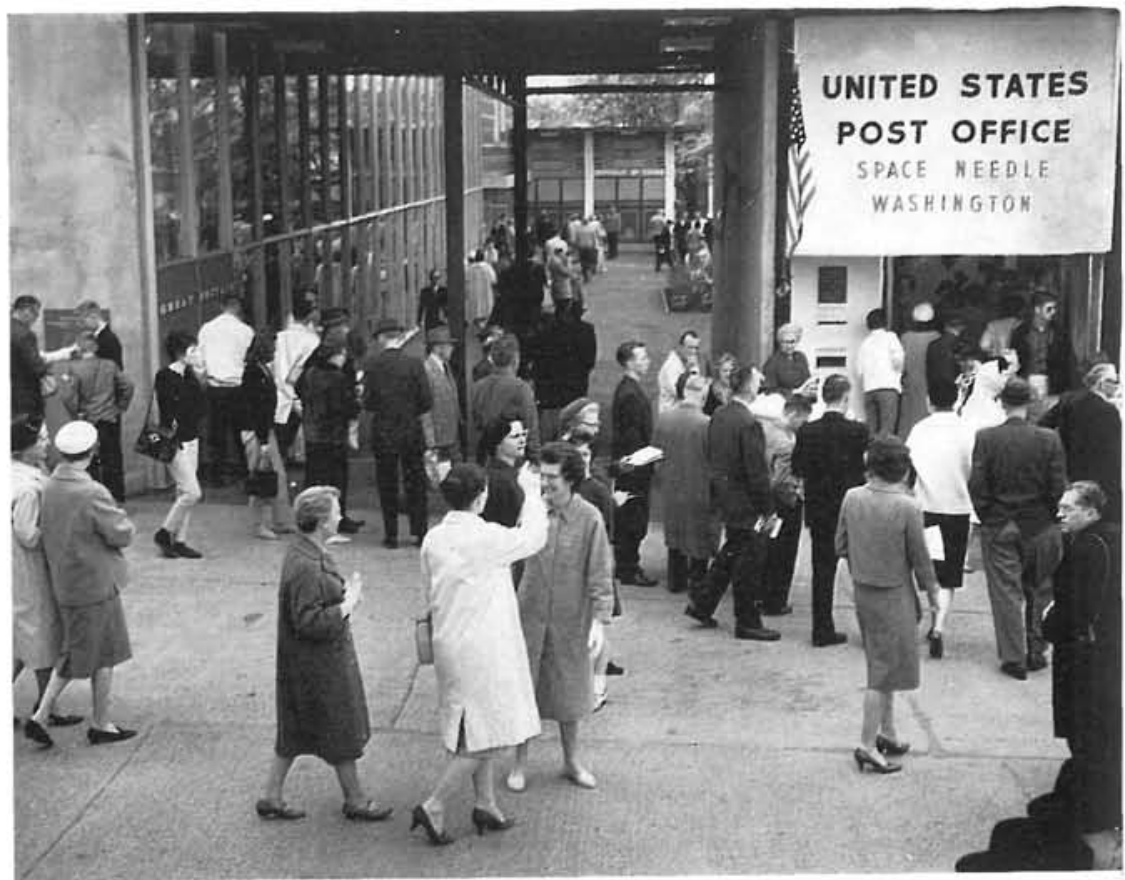

\section{Edificio pretensado para el estacionamiento de vehículos}

El Ayuntamiento ha habilitado un nuevo estacionamiento de vehículos, construido por la casa Norman G. Jacobson \& Associates, de Seattle.

El edificio consta de cuatro plantas, con una capacidad de 1.500 vehículos y un presupuesto aproximado de 1.000 dólares por jaula.

Con un tráfico de dirección única, pendientes máximas del 6 por $100 \mathrm{y}$ suficientes puertas de acceso, se puede llenar o desalojar el local en quince o veinte minutos.

La exposición es fascinante, y si se quieren contemplar bien todas las maravillas alli instaladas es necesario efectuar varias visitas.

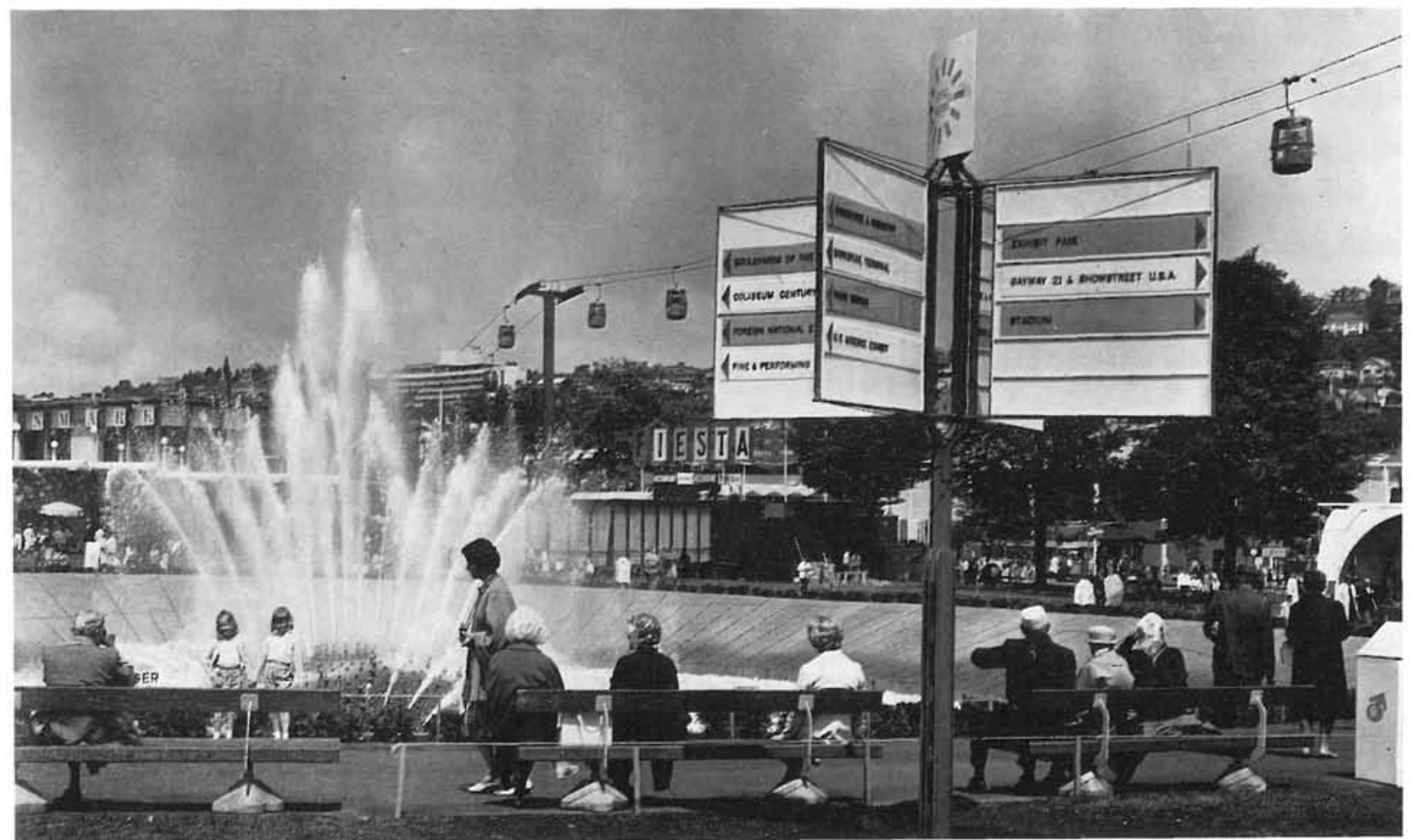




\section{L'exposition momalicale de Sertite}

Harlan H. Edwards, ingénieur.

L'ensemble d'édifices qui constituaient l'Exposition Mondiale de Seattle, dans la ville du même nom, aux Etats-Unis, a nécessité una longue période de préparation, car l'obtention des fonds indispensables pour couvrir les exigences d'un proje de telle envergure et d'une telle portée d'ordre scientifique et artistique, a dû être assurée par une aide économique, tant fédérale que de l'Etat et des institutions industrielles.

Chacun des édifices avait une signification et un motif qui le caractérisait et distinguait des autres. Dans l'ensemble on remarquait particulièrement pour sa hauteur l'Aiguille de l'Espace, dont la partie supérieure était un mirador surmonté d'un flambeau.

Un autre ouvrage artistique digne d'intérêt était la fontaine lumineuse, aux riches couleurs, remarquablement ornemental, dessinée par un groupe d'architectes japonais.

Le Pavillon Scientifique des Etats-Unis méritait une attention toute particulière, car, mis à part ses qualités purement architecturales, il abritait l'exposition des derniers progrès seientifiques, de telle façon qu'ils constituassent une vulgarisation d'assimilation facile pour les visiteurs en général.

Un théâtre avait été construit également pour les représentations d'opéra, concerts, ete.

L'ensemble des édifices de cette importante Exposition Ntait complété par une série d'installations pour le transport des visiteurs et le stationnement des voitures.

\section{The Semtrite World Exhthibition}

Harlan H. Edwards, engineer.

The group of buildings which constituted the so called Seattle World Exhibition, at Seattle, took a long time in designing and construction, due to the difficulties in obtaining the necessary funds for a project of such vast scientific and artistic scope. For its final accomplishment the cooperation of the local and federal governments and of private industries was essential.

Each of the buildings had a motive and quality which distinguished it from the others. The space needle stood out because of its heigth and the observation platform and burning torch at the top.

The luminous fountain was another notable feature. It was highly ornamental and an outstanding colour spectacle. It was designed by a group of Japanese architects.

The United States scientific pavillion merited special attention, for in addition to its formal significance, it housed the latest scientifie developments in a manner that made them easy to understand by the general public.

The Exhibition also included a theatre suitable for stage, opera and concert performances.

The project was completed with a number of auxiliary installations as well as transport and parking facilities for the visitors.

\section{Die Weltausstellung in Seattie}

Harland H. Edwards, Ingenieur.

Die Bebauung des Geländes für die Weltausstellung in Seattle, einer Stadt in den Vereinigten Staaten, hat eine sehr lange Vorgeschichte, denn die Bereitstellung von Mitteln für die Durchführung eines solch ehrgeizigen und umfassenden Projektes, sowohl auf wissenschaftlichem als auch künstlerischem Gebiet, bedurfte der finanziellen Unterstützung sowohl seitens des Staates als auch der Industrie.

Jeder einzelne Pavillon hat seine eigene Charakteristik und Bedeutung, durch die er sich von den anderen unterscheidet. Besonders zu erwähnen ist die «Weltraumnadel», die an Grösse die übrigen Bauten überragt und in ihrem Teil oberhalb des Kapitells gleichzeitig als Ausblickturm verwendet wird.

Ein weiteres Kunstwerk ist der reich verzierte Leuchtbrunnen von japanischen Architekten entworfen.

Besondere Aufmerksamkeit verdient der wissenschaftliche Pavillon der Vereinigten Staaten, einmal aufgrund seiner äusseren Form und zum anderem aufgrund der Art und Weise, wie man versucht, die allerneuesten technischen Errungensehaften auf einfache Weise dem Durchschnittsbesucher verständlich zu machen.

Ebenso hat man ein Theater zur Aufführung von Opern und Konzerten errichtet.

Ergänzt wird der ganze Ausstellungskomplex durch eine Reihe von Einrichtungen für den Transport von Besuchern und zum Parken der Wagen. 